\title{
Manejo de las redes sociales electrónicas por parte de los estudiantes de medicina: el caso de la publicación de fotografías de los pacientes y el profesionalismo médico
}

\author{
Pedro José Villamizar, Sandra Milena Moreno, Freddy Moreno \\ Facultad de Ciencias de la Salud, Pontificia Universidad Javeriana, Cali, Colombia
}

\begin{abstract}
Introducción. Se hizo la caracterización sociodemográfica de los estudiantes de Medicina de la Pontificia Universidad Javeriana en Cali, Colombia, y se recolectó información sobre el acceso a internet, el manejo de redes sociales electrónicas y la difusión de fotografías con los pacientes durante las prácticas de docencia asistencial, lo cual es una práctica inapropiada y contraria al profesionalismo médico.

Objetivo. Determinar el manejo de las redes sociales electrónicas por parte de los estudiantes de medicina, en cuanto a la publicación de fotografías con los pacientes.

Materiales y métodos. Se hizo un estudio descriptivo de corte transversal que caracterizó a los estudiantes de Medicina de la Pontificia Universidad Javeriana de Cali. De los 423 estudiantes matriculados en enero de 2013, 299 diligenciaron una encuesta diseñada con el programa Cardiff TELEform $^{\circledR}$, versión 10.0. Asimismo, se constituyó un grupo focal con 20 estudiantes escogidos aleatoriamente para discutir sobre la publicación de fotografías en las redes sociales electrónicas.

Resultados. El 97,6 \% de los estudiantes encuestados manifestó ser usuario activo de, por lo menos, una de las principales redes sociales electrónicas $(96,2 \%$ de Facebook, 70,5 \% de Instagram y 44,1\% de Twitter). El 17,6 \% (52) manifestó haber publicado, por lo menos, en una ocasión una fotografía tomada durante la atención a los pacientes.

Conclusión. La gran mayoría de los estudiantes tenía teléfonos inteligentes y había publicado su perfil de usuario en las principales redes sociales electrónicas, a través de las cuales difundían fotografías de los pacientes durante la atención médica asistencial, así como durante diversas actividades, lo que podría tener implicaciones éticas y legales, e ir en contra del profesionalismo médico.
\end{abstract}

Palabras clave: educación de pregrado en medicina, estudiantes de medicina, red social, profesionalismo médico, ética médica.

doi: http://dx.doi.org/10.7705/biomedica.v36i1.2646 Management of electronic social networks by students of medicine: The case of publication of
photographs with patients and professional medical conduct

Introduction: The socio-demographic characterization of medical students at the Pontificia Universidad Javeriana in Cali, Colombia, was made using a survey and focus group discussion to collect information on access to the Internet, management of electronic social networks and the dissemination of photographs with patients taken during medical teaching rounds, a practice that constitutes a breach of professional medical conduct.

Objective: To identify the management of social electronic networks by students of medicine with respect to the publication of photographs with patients.

Materials and methods: A descriptive cross-sectional study was undertaken to characterize students of medicine at the Pontificia Universidad Javeriana in Cali. Of the 423 students enrolled in January 2013, 299 participated in a survey designed with the software Cardiff TELEform ${ }^{\circledR}$, version 10.0. Twenty students were also selected at random for a focus group discussion on the publication of photographs with patients on electronic social networks.

Results: Of the students surveyed, $97.6 \%$ claimed to be active users of at least one of the main electronic social networks (96.2\% Facebook, 70.5\% Instagram and 44.1\% Twitter). Of these, 52 (17.6\%) admitted having published a photograph showing patient care on at least one occasion.

\section{Contribución de los autores:}

Todos los autores participaron en la revisión sistemática de la bibliografía, el diseño del proyecto de investigación, la obtención, el análisis y la discusión de los resultados, y la elaboración y revisión del manuscrito. 
Conclusion: Most of the students had smartphones and user profiles on the main social electronic networks, on which they disseminated photographs of patients taken during teaching rounds, as well as images of various activities which could have ethical and legal implications and contravene standards of professional medical conduct.

Key words: Education, medical, undergraduate; students, medical; social networking; ethics, medical. doi: http://dx.doi.org/10.7705/biomedica.v36i1.2646

En la primera década del siglo XXI, el uso de las redes sociales a través de internet transformó las prácticas sociales de las personas e instituciones. Las redes sociales constituyen un medio social virtual que permite a los seres humanos establecer relaciones por medio de un conjunto de redes de comunicación interconectadas entre sí $(1,2)$.

Entre las redes sociales electrónicas más reconocidas se encuentran Facebook, Instagram, Twitter, MySpace, YouTube y Linkedin, en cuyas plataformas virtuales los usuarios pueden compartir mensajes de texto, fotografías, vídeos y accesos directos a diversas fuentes de información disponibles en internet $(3,4)$, en una red compleja que cuenta con cerca de mil millones de personas, lo que ha transformado la forma en que las personas interpretan la ética de sus relaciones con amigos, compañeros de trabajo, padres, hijos, empleadores, empleados, profesores, estudiantes, vendedores, compradores e, inclusive, las relaciones entre médicos y pacientes $(5,6)$.

Si bien el propósito inicial de cada red social electrónica era funcionar como una herramienta de conexión de los seres humanos con sus conocidos y con otras personas de su mismo entorno, los usuarios comenzaron a expresarse en ámbitos psicológicos y afectivos que pueden tener implicaciones éticas y legales. En este sentido cabe mencionar la publicación de datos, fotos y vídeos que violan la privacidad de otras personas y las suplantan creando perfiles falsos y violando los derechos de autor con descargas ilegales y la obtención o intercambio de contenidos (7-9).

El estudio de las implicaciones éticas y legales del emergente fenómeno de las redes sociales electrónicas, se centra en los patrones y efectos del uso y la integración de los dispositivos

\footnotetext{
Correspondencia

Freddy Moreno, Facultad de Ciencias de la Salud, Pontificia Universidad Javeriana, Calle $18 \mathrm{~N}^{\circ} 118-250$, Cali, Colombia Teléfono: (052) 3218200 , extensión 8927

fmorenog@javerianacali.edu.co

Recibido: 15/12/14; aceptado: 08/09/15
}

electrónicos y de las llamadas nuevas tecnologías de la información y la comunicación en muchas esferas de la vida cotidiana, lo que afecta significativamente las relaciones humanas en la familia, en la comunidad, en las instituciones educativas y en las empresas prestadoras de servicios en salud, por citar las de mayor impacto sociológico. Dicho estudio constituye hoy una rama de la ética aplicada denominada "ética informática" o "ciberética", la cual evalúa y analiza el impacto social y ético de las citadas tecnologías (10).

La discusión se ha centrado en el manejo de la privacidad en las redes sociales electrónicas a partir de las buenas prácticas de control de la información en términos de acceso e integridad contextual $(11,12)$. Si bien es cierto que los administradores de estas redes manifiestan explícitamente que el material publicado es de total responsabilidad de los usuarios, los mecanismos para verificar si se ha vulnerado el derecho a la privacidad, o se ha incurrido en prácticas inadecuadas desde el punto de vista ético, no resultan contundentes. Además, ante lo atractivo y útil que puede resultar el libre acceso a toda la información, los usuarios no comprenden o minimizan las consecuencias de compartir e intercambiar información en las redes sociales, lo cual se ha interpretado como la aceptación social de la pérdida de intimidad y la ausencia de límites que determinen qué tan público o privado debe ser un perfil de usuario en los entornos virtuales y cómo se debe enlazar con la vida real $(13,14)$.

Con respecto al uso de las redes sociales electrónicas por parte de los estudiantes, residentes y profesionales de la medicina, las aplicaciones en los teléfonos móviles inteligentes y el acceso permanente a internet se han convertido en el mecanismo más rápido de intercambio de información personal y profesional mediante la publicación de comentarios y fotografías, lo cual puede catalogarse como una conducta inapropiada que compromete el profesionalismo y la ética médica, principalmente cuando se vulnera la privacidad y la confidencialidad de la información sobre los pacientes (15-17). 
Este tipo de divulgación genera una serie de inconvenientes médicos y legales por mala práctica y responsabilidad profesional para las personas, las instituciones educativas y las empresas prestadoras de servicios de salud con las cuales se establecen convenios docente-asistenciales. Tales inconvenientes se inscriben en el ámbito de la vulneración de la confidencialidad de la historia clínica en lo legal, la identificación de los pacientes en lo ético y la violación del secreto médico en lo profesional (18-20). Así pues, si bien el desarrollo y el uso de las redes han generado un cambio de paradigma en la comunicación entre médico y paciente, médico e institución, y paciente e institución, también pueden vulnerar los principios del profesionalismo en la práctica médica (21).

El profesionalismo, que incluye entre otros aspectos el respeto por la privacidad de los pacientes (22-24), constituye una de las seis competencias básicas que deben desarrollar y mantener los estudiantes de Medicina durante el pregrado y la residencia, y los médicos en su ejercicio profesional. Hoy el uso masivo y poco controlado de las redes sociales electrónicas ha provocado un debate ético en este campo $(25,26)$, pues los estudiantes y los profesionales de la Medicina utilizan estas redes para relacionarse con sus colegas y con sus pacientes, y como una herramienta médica que trasciende su uso exclusivo en el sitio de trabajo, lo cual si bien resulta beneficioso, los expone al riesgo constante de cruzar el límite entre lo que se considera un comportamiento profesional y lo que no. Al igual que cualquier ciudadano, los profesionales de la Medicina tienen la libertad y el derecho de usar las redes sociales, pero deben tener especial cuidado para no trasgredir las normas éticas que rigen la profesión médica (27).

En este sentido, las asociaciones médicas y los colegios de médicos en diversos países han propuesto guías y manuales para reglamentar el comportamiento de los médicos en las redes sociales electrónicas (28-31). Entre las normas adoptadas, se ha planteado que la publicación de información y de fotografías de los pacientes tomadas durante la consulta, constituye una franca vulneración del derecho a la intimidad y a la confidencialidad de la información contenida en la historia clínica, lo cual tiene implicaciones éticas y legales y afecta negativamente la confianza de las personas en la profesión médica (19).
En este contexto, en el presente estudio se propuso analizar el manejo que los estudiantes de Medicina de la Pontificia Universidad Javeriana de Cali hacen de las redes sociales, específicamente en lo relacionado con la publicación de fotografías de los pacientes y el profesionalismo médico.

\section{Materiales y métodos}

Se hizo un estudio descriptivo de corte transversal para analizar el manejo de las redes sociales electrónicas, específicamente la publicación de fotografías con los pacientes y el profesionalismo médico, a partir de la caracterización sociodemográfica de los estudiantes de Medicina de la Facultad de Ciencias de la Salud de la Pontificia Universidad Javeriana de Cali (Colombia). No se tuvieron en cuenta los estudiantes de primer semestre, ya que este no había comenzado.

Los datos se recolectaron mediante una encuesta diligenciada por los participantes y estructurada con base en los siguientes dominios: 1) sociodemográfico y cultural; 2) académico y cognitivo; 3) socioafectivo y sexual; 4) ético, moral y religioso; 5) participativo y gremial; 6) político e ideológico; 7) relativo a la salud y el uso del tiempo libre, y 8) deportivo. Estos dominios se habían empleado en un estudio previo de caracterización del estudiante javeriano (32), de cuya información solo se utilizó la relacionada con el uso de las redes. Antes de la encuesta los estudiantes no recibieron ningún tipo de información adicional sobre el manejo de estas redes y el profesionalismo médico diferente a la información incluida en el plan de estudios en asignaturas como la de medicina basada en la etiqueta y la de bioética.

La encuesta se diseñó en el programa Cardiff TELEform $^{\circledR}$, versión 10.0, que permite la captura automática de los datos a partir de las respuestas al cuestionario mediante el sistema de reconocimiento óptico de caracteres con un lector óptico. Además, el programa consolida automáticamente la información de las encuestas y la exporta a una base de datos compatible con el programa IBM SPSS Statistics ${ }^{\circledR}$, versión 21.0, con el cual se analizan las frecuencias.

Por otra parte, se seleccionaron aleatoriamente 20 estudiantes con quienes se constituyó un grupo focal para recolectar información mediante una entrevista grupal semiestructurada $(33,34)$ en torno al acceso y el manejo de la información en las redes sociales más populares (Facebook, Instagram y Twitter), y con la cual se obtuvo información sobre 
las prácticas, creencias, actitudes, sentimientos, experiencias y reacciones de los participantes, con el fin de determinar la realidad sobre el uso de dichas redes.

\section{Consideraciones éticas}

Una vez el estudio fue aprobado por el Comité de Ética e Investigación en Humanos de la Pontificia Universidad Javeriana de Cali, los estudiantes contestaron la encuesta tras aceptar su participación en el estudio y firmar el consentimiento informado. En todo momento se preservó la confidencialidad de la información de acuerdo con la Resolución 008430 del Ministerio de la Protección Social (35) y con la Declaración de Helsinki (36).

\section{Resultados}

De los 423 estudiantes matriculados en el programa de Medicina en el primer período (enerojunio) de 2013, 299 (70,7 \%) de los inscritos entre el segundo y el octavo semestres contestaron la encuesta; 64,3 \% correspondía a mujeres $y, 35,7 \%$, a hombres; 90,9 \% era mayor de 18 años, aunque al ingresar al plan de estudios en el primer semestre, 43,7 \% era menor de 18 años. En el momento de contestar la encuesta la edad mínima de los participantes era de 16 años y, la máxima, de 28 (media de 19,79; mediana de 20,00; desviación estándar de 1,980 y varianza de 3,921). Con respecto al estrato socioeconómico de los estudiantes residentes en la ciudad de Cali, 69,9\% habitaba viviendas de estratos 4,5 y 6 , y $9,4 \%$, de los estratos 1,2 y 3 , en tanto que $20,7 \%$ no sabía cuál era el estrato en el momento de responder la encuesta. Todos los estudiantes contaban en sus viviendas con los servicios públicos de energía, agua, alcantarillado y recolección de basuras. En cuanto a los medios de comunicación disponibles en el lugar de residencia, todos los estudiantes tenían servicio de telefonía fija y telefonía celular, televisión e internet, y otros contaban con telefonía satelital y radioteléfono (37\%).

Del total de participantes, 292 (97,6 \%) manifestaron ser usuarios activos de, por lo menos, una de las principales redes sociales: 281 (96,2 \%) de Facebook, 206 (70,5 \%) de Instagram y $129(44,1 \%)$ de Twitter; 149 (51\%) estudiantes tenían un perfil de usuario en las tres redes sociales mencionadas.

De los 292 estudiantes que tenían un perfil de usuario en alguna de las redes sociales, 213 (73 \%) contaban con teléfonos inteligentes y acceso directo a ellas, 35 (12 \%) accedían a través de dispositivos con conexión a internet inalámbrica como tabletas electrónicas, y 44 (15\%) lo hacían a través de computadores portátiles y de oficina. El 17,8 \% de los usuarios de las redes sociales tenía un perfil privado y, el resto, uno público con algunas restricciones.

De los 213 estudiantes poseedores de teléfonos inteligentes, 210 (98,6 \%) se mantenían conectados en tiempo real permanentemente, de tal forma que accedían a las redes sociales cada vez que recibían una notificación automática. Los tres $(1,4 \%)$ estudiantes restantes accedían digitando su usuario y contraseña máximo dos veces al día, principalmente al iniciar y al finalizar el día.

Del total de estudiantes, 190 (65,1 \%) manifestaron que publicaban habitualmente información escrita (mensajes de texto) o gráfica (fotografías y vídeos) con el tipo de mensaje propio de cada red social y de acuerdo con las capacidades técnicas de cada sistema.

Entre los participantes, 52 (17,6 \%) manifestaron haber publicado, por lo menos, en una ocasión una fotografía tomada durante la atención a pacientes; 46 (88 \%) de estas publicaciones fueron hechas por estudiantes de sexto a octavo semestre que habían comenzado sus rotaciones en las diferentes instituciones prestadoras de servicios de salud; las seis (12\%) publicaciones restantes correspondían a actividades extramurales durante las prácticas de salud pública. Por otra parte, 263 (87,9\%) estudiantes manifestaron saber que la publicación de fotografías tomadas durante la atención a los pacientes en las que fuera posible identificarlos, constituía una mala práctica profesional con implicaciones éticas y legales; entre los estudiantes con cuenta de usuario en alguna de las redes, 17 (5,8 \%) habían hecho este tipo de publicaciones, principalmente en Facebook e Instagram, aun sabiendo las implicaciones legales y éticas.

Por otro lado, 285 (95,3 \%) estudiantes manifestaron que habían publicado información que de una u otra forma podría catalogarse como poco profesional, fundamentalmente en forma de comentarios y fotografías de actividades sociales y culturales que, tomadas fuera de contexto, podían interpretarse como situaciones de intoxicación por consumo de alcohol, o imágenes de contenido sexual explícito o de consumo de cigarrillo, así como la expresión de posiciones personales frente a problemas médicos y sociales actuales, como el aborto, la eutanasia, el matrimonio y la adopción por parte de parejas homosexuales y el consumo 
de marihuana como método terapéutico, entre otras, las cuales, por ser controversiales, podían interpretarse como poco profesionales por parte de personas del común y de pacientes con acceso a los perfiles de usuario.

En el grupo focal, los estudiantes manifestaron que efectivamente habían publicado fotografías de ellos mismos durante la atención a pacientes en los sitios de práctica, principalmente en Facebook e Instagram. Al discutir sobre el propósito de hacer este tipo de publicaciones, las respuestas casi unánimes fueron que "estoy demostrando a familiares y amigos que como médicos en formación ya estamos en contacto con los pacientes" y que "tengo sentido de pertenencia por la profesión y por la universidad". Cuando se les preguntó si reconocían que dicha práctica no era profesional ni ética, la mayoría dijo que "no percibo nada de malo en subir este tipo de fotografías", "no estamos haciendo nada de malo" y "todos lo hacen sin ningún problema, incluso médicos". De igual forma, los estudiantes manifestaron que las fotografías y su publicación habían sido autorizadas verbalmente por los pacientes y, en el caso de los niños, por los padres y acudientes, por lo que al preguntárseles si había algún asentimiento o consentimiento informado anexado en la historia clínica, la respuesta fue negativa.

Frente a la pregunta de si sabían que la publicación de fotografías con los pacientes en las redes sociales tenía implicaciones éticas y legales, las respuestas incluyeron "no sé", "no creo" y "sí, he leído algo al respecto", pero incluso quienes dijeron tener conocimiento de ello, habían publicado fotografías argumentando que "no hay ninguna mala intención".

Con respecto al manejo poco profesional de la información, hubo consenso sobre el derecho a la libre expresión y a la separación entre la vida cotidiana y el ámbito profesional; en este contexto, se registraron expresiones como "yo hago con mi tiempo libre lo que quiera", "yo puedo publicar mis actividades personales en mi perfil de usuario sin que esto afecte mi imagen profesional" y "no creo que actividades como fumar cigarrillo o tomarme unos tragos afecte mi profesionalismo".

\section{Discusión}

El profesionalismo es una competencia fundamental para la obtención y el mantenimiento de la licencia de médico, y la certificación y renovación del certificado de la especialización médica en muchos países. No obstante, las personas divulgan información sobre su vida personal sin someterse al escrutinio profesional, lo que desemboca en la publicación imprudente de opiniones discriminatorias y en la expresión de conductas sexuales inapropiadas o estados de intoxicación por alcohol que reflejan un perfil individual incompatible con los requisitos de ingreso a las instituciones, las cuales consideran esto como una conducta falta de profesionalismo $(22,23,37,38)$. El punto de quiebre radica en que la mayoría de los estudios concluyen que entre los estudiantes de Medicina aspirantes a una residencia y los educadores médicos no existe un consenso sobre lo que se considera una publicación imprudente, inadecuada o poco profesional (17), por lo que esgrimen el argumento de no ser conscientes de que las publicaciones en las redes sociales electrónicas pueden reflejar una falta de profesionalismo médico (19).

Sin embargo, y pese a que el uso no profesional de estas redes por parte de médicos se encuentra muy bien documentado, y que las sociedades profesionales han elaborado manuales de comportamiento y manejo ético y profesional de los perfiles de usuario, no se han desarrollado mecanismos para detectar estas conductas médicas (39), aun cuando se trata de una comunidad profesional que ejerce la regulación de las conductas de sus integrantes (comités de ética médica, asociaciones de especialistas, cuerpos colegiados, asociaciones académicas, etc.) y en la que todos deben velar por el cumplimiento de las normas éticas y profesionales, inclusive en el escenario virtual de las redes sociales (40-43).

Según los resultados de la discusión del grupo focal, esta se centró en el desconocimiento de los estudiantes de las implicaciones éticas y legales de la publicación de fotografías de los pacientes en las redes, lo cual indudablemente es inapropiado e imprudente, antiprofesional y antiético, y resulta en una vulneración del derecho a la privacidad de los pacientes, a la confidencialidad de su historia clínica y al secreto médico. Sin embargo, los estudiantes manifestaron su desacuerdo sobre lo que se puede considerar e interpretar como inapropiado y poco profesional en el ejercicio de la Medicina cuando se trata de la divulgación a través de las redes sociales de hechos de su vida privada. De todas maneras, se percibió cierto grado de conciencia de que los profesores, las directivas y los mismos pacientes de una u otra forma tienen acceso a los perfiles de usuarios y 
que los comentarios y las fotografías inapropiadas e imprudentes que vulneran la confidencialidad de la información sobre los pacientes pueden generar expectativas negativas sobre el comportamiento ético y profesional del médico en formación, lo que finalmente afectará también la imagen de la institución de educación superior y de la institución prestadora de servicios.

En este sentido, se discutió la Ley 23 de 1981 (44) en la cual se estipula que la historia clínica "es un documento privado sometido a reserva que únicamente puede ser conocido por terceros previa autorización del paciente" y que "el médico está obligado a guardar el secreto profesional en todo aquello que por razón del ejercicio de su profesión haya visto, oído o comprendido, salvo en los casos contemplados por disposiciones legales"; así como la Resolución 1995 de 1999 (45) que determina que la historia clínica "es un documento privado, obligatorio y sometido a reserva, en el cual se registran cronológicamente las condiciones de salud del paciente, los actos médicos y los demás procedimientos ejecutados por el equipo de salud que interviene en su atención". Por lo tanto, las fotografías tomadas a los pacientes, incluso si estos las han autorizado, hacen parte de la historia clínica y de los documentos legales, técnicos, científicos y administrativos pertinentes en los procesos de atención y, por ende, quedan cobijadas por la citada legislación.

Asimismo, se dejó claro que la intimidad, la privacidad y la confidencialidad constituyen derechos fundamentales de los seres humanos, adoptados por la Asamblea General de la Organización de las Naciones Unidas y ratificados por la Corte Interamericana de Derechos Humanos, a la cual Colombia se encuentra adscrita, y protegidos por los principios de la Asociación Médica Mundial, incluida la Declaración de Helsinki, y por el estado colombiano en sus leyes, empezando por la Constitución Política.

Al comparar los resultados de este estudio con otros reportes (cuadro 1) hallados en la revisión sistemática de la literatura a través de PubMed, quedó claro que la investigación de las implicaciones éticas y legales del manejo de la información en las redes sociales por parte de estudiantes y residentes de Medicina y de profesionales médicos, se centra en la vulneración del derecho a la confidencialidad de los pacientes y en el detrimento del profesionalismo médico.
Aunque este estudio tiene limitaciones porque no valoró directamente los perfiles de usuario de los estudiantes en las redes sociales, con la encuesta y la discusión en el grupo focal se pudo tener una percepción sobre lo que representa para los estudiantes la publicación de fotografías de los pacientes durante su atención.

La encuestas y la discusión del grupo focal permitieron determinar que la mayoría de los estudiantes desconoce las implicaciones éticas y legales de la publicación y difusión de fotografías que vulneren la confidencialidad de la atención médica de los pacientes, y que no logran determinar el punto en el que las publicaciones y los comentarios sobre sus actividades socioculturales pueden resultar controversiales en nuestra sociedad y ser juzgadas fuera de contexto como poco profesionales por personas del común, así como por los pacientes y por los integrantes de la comunidad académica, científica y asistencial que, de una u otra forma, tienen acceso a los perfiles de usuario.

En conclusión, la muestra estudiada correspondía a una población homogénea, con las mismas características sociodemográficas y de acceso a teléfonos celulares inteligentes que les permitían mantenerse conectados en tiempo real a las redes, principalmente Facebook, y acceder a los comentarios y fotografías publicadas por ellos mismos y por sus contactos. Asimismo, una cantidad significativa de estudiantes había publicado en las redes sociales fotografías tomadas en los sitios de docencia asistencial, en diferentes instituciones prestadoras de servicios de salud y durante la atención a los pacientes. De igual forma, los estudiantes manifestaron que publicaban contenidos que podrían ser catalogados como poco profesionales. Las redes sociales preferidas para este tipo de publicaciones eran Facebook e Instagram.

La recomendación, entonces, es que frente a la tendencia a publicar fotografías que vulneran la confidencialidad de la historia clínica, permiten conocer la identidad de los pacientes, de las instituciones académicas y de los prestadores de servicios de salud, y revelan el secreto médico, se incluyan en las cátedras de Ética Médica y Bioética, y en cualquier espacio académico afín, conferencias y clases magistrales que propicien la discusión sobre las implicaciones éticas y legales de la difusión de fotos de los pacientes en las redes sociales. Lo mismo aplica para las actividades socioculturales que pueden comprometer 
Cuadro 1. Estudios sobre el uso no profesional de las redes sociales electrónicas por parte de estudiantes y profesionales de Medicina

\begin{tabular}{|c|c|c|c|c|c|c|}
\hline Autores & Año & Lugar & $\begin{array}{l}\text { Tipo de } \\
\text { estudio }\end{array}$ & Muestra & Objetivo & Resultados y conclusiones \\
\hline $\begin{array}{l}\text { Thompson, } \\
\text { et al. (15) }\end{array}$ & 2008 & $\begin{array}{l}\text { Estados } \\
\text { Unidos }\end{array}$ & Descriptivo & $\begin{array}{l}\text { Estudiantes } \\
\text { de Medicina } \\
\text { y residentes }\end{array}$ & $\begin{array}{l}\text { Determinar el uso } \\
\text { no profesional de } \\
\text { Facebook }\end{array}$ & $\begin{array}{l}\text { El } 44,5 \% \text { contaba con perfil de usuario, } \\
37,5 \% \text { manejaba perfiles privados, } \\
64,3 \% \text { publicaba mensajes de texto, } \\
\text { enlaces y fotografías con gran frecuencia. }\end{array}$ \\
\hline $\begin{array}{l}\text { Chretien, } \\
\text { et al. (19) }\end{array}$ & 2009 & $\begin{array}{l}\text { Estados } \\
\text { Unidos }\end{array}$ & Descriptivo & $\begin{array}{l}\text { Estudiantes } \\
\text { de Medicina }\end{array}$ & $\begin{array}{l}\text { Determinar el uso } \\
\text { no profesional } \\
\text { de las RSE }\end{array}$ & $\begin{array}{l}\text { El } 52 \% \text { de los participantes publicaba } \\
\text { mensajes con lenguaje obsceno, } 48 \% \\
\text { usaba expresiones discriminatorias, } 38 \% \\
\text { incluía representaciones textuales y gráficas } \\
\text { sugestivas de contenido sexual, } 39 \% \text { aludía } \\
\text { al consumo e intoxicación por alcohol y } \\
\text { sustancias ilícitas. }\end{array}$ \\
\hline $\begin{array}{l}\text { Landman, } \\
\text { et al. (21) }\end{array}$ & 2010 & $\begin{array}{l}\text { Estados } \\
\text { Unidos }\end{array}$ & Descriptivo & $\begin{array}{l}\text { Residentes y } \\
\text { profesores de } \\
\text { Medicina }\end{array}$ & $\begin{array}{l}\text { Determinar el uso } \\
\text { no profesional } \\
\text { de Facebook }\end{array}$ & $\begin{array}{l}\text { El } 64 \% \text { de los residentes y } 22 \% \text { de los } \\
\text { profesores contaban con perfil de usuario } \\
\text { en Facebook, } 50 \% \text { de los perfiles era } \\
\text { público, } 31 \% \text { de las publicaciones se } \\
\text { relacionaba con la práctica médica, } 14 \% \\
\text { vulneraba la privacidad de los pacientes. }\end{array}$ \\
\hline $\begin{array}{l}\text { Chretien, } \\
\text { et al. (37) }\end{array}$ & 2011 & $\begin{array}{l}\text { Estados } \\
\text { Unidos }\end{array}$ & Descriptivo & $\begin{array}{l}\text { Profesionales } \\
\text { de Medicina }\end{array}$ & $\begin{array}{l}\text { Determinar el uso } \\
\text { no profesional } \\
\text { de Twitter }\end{array}$ & $\begin{array}{l}\text { Se evidenció la vulneración de la } \\
\text { confidencialidad de la información de } \\
\text { los pacientes en los perfiles de usuario } \\
\text { de Twitter. }\end{array}$ \\
\hline $\begin{array}{l}\text { Greysen, } \\
\text { et al. (40) }\end{array}$ & 2012 & $\begin{array}{l}\text { Estados } \\
\text { Unidos }\end{array}$ & $\begin{array}{l}\text { Descriptivo } \\
\text { retrospectivo }\end{array}$ & $\begin{array}{l}\text { Profesionales } \\
\text { de Medicina }\end{array}$ & $\begin{array}{l}\text { Evidenciar denuncias } \\
\text { por conductas no } \\
\text { apropiadas en } \\
\text { las redes sociales } \\
\text { electrónicas que } \\
\text { condicionaran el } \\
\text { profesionalismo } \\
\text { de los médicos }\end{array}$ & $\begin{array}{l}\text { El } 92 \% \text { de los participantes había vulnerado } \\
\text { el derecho a la confidencialidad de la } \\
\text { información de los pacientes y la } \\
\text { prescripción de medicamentos sin una } \\
\text { relación clínica establecida. }\end{array}$ \\
\hline $\begin{array}{l}\text { von } \\
\text { Muhlen M, } \\
\text { et al. (46) }\end{array}$ & 2012 & Suecia & $\begin{array}{l}\text { Revisión } \\
\text { sistemática de } \\
\text { la literatura }\end{array}$ & $\begin{array}{l}\text { Estudiantes y } \\
\text { profesionales } \\
\text { de Medicina, } \\
\text { Odontología, } \\
\text { Enfermería y } \\
\text { Farmacéutica }\end{array}$ & $\begin{array}{l}\text { Determinar el uso } \\
\text { de Facebook } \\
\text { y Twitter }\end{array}$ & $\begin{array}{l}\text { Entre } 4 \text { y } 68,2 \% \text { de los participantes } \\
\text { publicaba contenidos que vulneraban el } \\
\text { derecho a la confidencialidad del paciente. }\end{array}$ \\
\hline $\begin{array}{l}\text { Golden, } \\
\text { et al. (25) }\end{array}$ & 2012 & $\begin{array}{l}\text { Estados } \\
\text { Unidos }\end{array}$ & Descriptivo & $\begin{array}{l}\text { Residentes de } \\
\text { Otorrinolaringología }\end{array}$ & $\begin{array}{l}\text { Evaluar el uso } \\
\text { de Facebook }\end{array}$ & $\begin{array}{l}\text { El } 51 \% \text { de los perfiles de usuario } \\
\text { era totalmente público, y } 11 \% \text { de } \\
\text { las publicaciones se consideraron } \\
\text { potencialmente violatorias de la ética } \\
\text { y del profesionalismo médico. }\end{array}$ \\
\hline $\begin{array}{l}\text { Farooqi, } \\
\text { et al. (47) }\end{array}$ & 2013 & Pakistán & Descriptivo & $\begin{array}{l}\text { Estudiantes } \\
\text { de Medicina }\end{array}$ & $\begin{array}{l}\text { Evaluar el efecto } \\
\text { de Facebook en } \\
\text { la vida social }\end{array}$ & $\begin{array}{l}\text { El } 64 \% \text { de los participantes tenía perfil de } \\
\text { usuario, } 40,1 \% \text { revisaba las notificaciones } \\
\text { de su cuenta tres veces al día como mínimo, } \\
\text { sin importar la actividad que se encontrara } \\
\text { haciendo. }\end{array}$ \\
\hline $\begin{array}{l}\text { Brynolf, } \\
\text { et al. (48) }\end{array}$ & 2013 & Suecia & Descriptivo & $\begin{array}{l}\text { Estudiantes y } \\
\text { profesionales } \\
\text { de Medicina }\end{array}$ & $\begin{array}{l}\text { Evaluar el contenido } \\
\text { de los mensajes } \\
\text { de Twitter }\end{array}$ & $\begin{array}{l}\text { El } 1,9 \% \text { se consideró como publicaciones } \\
\text { poco profesionales y } 0,2 \% \text {, como } \\
\text { potencialmente violatorias de la privacidad } \\
\text { de los pacientes. }\end{array}$ \\
\hline $\begin{array}{l}\text { Ponce, } \\
\text { et al. (26) }\end{array}$ & 2013 & $\begin{array}{l}\text { Estados } \\
\text { Unidos }\end{array}$ & Descriptivo & $\begin{array}{l}\text { Médicos que } \\
\text { solicitaron } \\
\text { residencias }\end{array}$ & $\begin{array}{l}\text { Evaluar el uso } \\
\text { de Facebook }\end{array}$ & $\begin{array}{l}\text { El } 9 \% \text { tenía contenidos que vulneraban la } \\
\text { ética médica debido a comportamientos poco } \\
\text { profesionales. }\end{array}$ \\
\hline $\begin{array}{l}\text { Clyde, } \\
\text { et al. (39) }\end{array}$ & 2014 & $\begin{array}{l}\text { Estados } \\
\text { Unidos }\end{array}$ & Descriptivo & $\begin{array}{l}\text { Estudiantes } \\
\text { de Medicina }\end{array}$ & $\begin{array}{l}\text { Evaluar el uso } \\
\text { de Facebook }\end{array}$ & $\begin{array}{l}\text { La mayoría de publicaciones sobre } \\
\text { situaciones personales y cotidianas se } \\
\text { podían interpretar como poco profesionales. }\end{array}$ \\
\hline $\begin{array}{l}\text { Este } \\
\text { estudio }\end{array}$ & 2014 & Colombia & Descriptivo & $\begin{array}{l}\text { Estudiantes } \\
\text { de Medicina }\end{array}$ & $\begin{array}{l}\text { Evaluar el uso de } \\
\text { Facebook, Twitter } \\
\text { e Instagram }\end{array}$ & $\begin{array}{l}\text { El } 17,6 \% \text { de los participantes manifestó } \\
\text { haber publicado por lo menos en una ocasión } \\
\text { una fotografía tomada durante la atención a } \\
\text { los pacientes; } 95,3 \% \text { publicaba información } \\
\text { que de una u otra forma podría catalogarse } \\
\text { como poco profesional, fundamentalmente } \\
\text { comentarios y fotografías relacionadas con } \\
\text { actividades socioculturales. }\end{array}$ \\
\hline
\end{tabular}


el profesionalismo de los estudiantes, residentes y profesionales de la medicina, a quienes se les sugiere leer y seguir las recomendaciones que al respecto propone la Asociación Médica Mundial.

\section{Conflicto de intereses}

Los autores del artículo hacen constar que no existe ningún tipo de conflicto de intereses que pueda poner en peligro la validez del estudio.

\section{Financiación}

Este artículo original deriva del proyecto de investigación "Caracterización sociodemográfica y manejo del tiempo libre de la población estudiantil de la carrera de Medicina de la Facultad de Ciencias de la Salud de la Pontificia Universidad Javeriana - Cali 2013", el cual fue financiado con recursos de la convocatoria interna 2013-2014 de la Pontificia Universidad Javeriana de Cali, Colombia.

\section{Referencias}

1. Centola D. The spread of behavior in an online social network experiment. Science. 2010;329:1194-7. http://dx. doi.org/10.1126/science. 1185231

2. Ugander J, Backstrom L, Marlow C, Kleinberg J. Structural diversity in social contagion. Proc Natl Acad Sci USA. 2012;109:5962-6. http://dx.doi.org/10.1073/pnas. 1116502109

3. Boyd DM, Ellison NB. Social network sites: Definition, history and scholarship. J Comput Mediat Commun. 2008;13:210-30. http://dx.doi.org/10.1111/j.1083-6101.2007. 00393.x

4. Black EW, Thompson LA, Duff WP, Dawson K, Saliba H, Paradise NM. Revisiting social network utilization by physicians-in-training. J Grad Med Educ. 2010;2:289-93. http://dx.doi.org/10.4300/JGME-D-10-00011.1

5. Wilson RE, Gosling SD, Graham LT. A review of Facebook research in the social sciences. Perspect Psychol Sci. 2012;7:203-20. http://dx.doi.org/10.1177/ 1745691612442904

6. Liu J-G, Ren Z-M, Guo Q, Chen D-B. Evolution characteristics of the network core in the Facebook PLoS ONE. 2014;9:1-7. http://dx.doi.org/10.1371/journal. pone. 0104028

7. Onnela JP, Reed-Tsochas F. Spontaneous emergence of social influence in online systems. Proc Natl Acad Sci USA. 2010;107:18375-80. http://dx.doi.org/10.1073/ pnas.0914572107

8. Bugeja MJ. Facing the Facebook. The Chronicle of Higher Education. January 23, 2006. Fecha de consulta: 8 de septiembre de 2014. Disponible en: http://chronicle.com/ article/Facing-the-Facebook/46904.

9. Dogruer N, Menevis I, Eyyam R. What is the motivation for using Facebook? Procedia - Social and Behavioral Sciences. 2011;15:2642-6. http://dx.doi.org/doi:10.1016/j. sbspro.2011.04.162
10. Gotterbarn D. The use and abuse of computer ethics. Journal of Systems and Software. 1992;17:75-80. http:// dx.doi.org/10.1016/0164-1212(92)90083-V

11. Bynum T. Computer and information ethics. En: Zalta EN, editor. The Stanford Encyclopedia of Philosophy. Palo Alto: Standford University; 2011. Fecha de consulta: 8 de septiembre de 2014. Disponible en: http://plato.stanford. edu/archives/spr2011/entries/ethics-computer/.

12. Nissenbaum M. Privacy as contextual integrity. Washington Law Review. 2004;79:119-57.

13. Boyd D, Hargittai E. Facebook privacy settings: Who cares? First Monday. 2010;15:13-20.

14. Spinello RA. Privacy and social networking technology. International Review of Information Ethics. 2011;16:41-6.

15. Thompson LA, Dawson K, Ferdig R, Black EW, Boyer $\mathrm{J}$, Coutts $\mathrm{J}$, et al. The intersection of online social networking with medical professionalism. J Gen Intern Med. 2008;23:954-7. http://dx.doi.org/10.1007/s11606-0080810-y

16. Lagu T, Kaufman EJ, Asch DA, Armstrong K. Content of weblogs written by health professionals. J Gen Intern Med. 2008;23:1642-6. http://dx.doi.org/10.1007/s11606008-0726-6

17. Go PH, Klaassen Z, Chamberlain RS. Attitudes and practices of surgery residency program directors toward the use of social networking profiles to select residency candidates: A nationwide survey analysis. J Surg Educ. 2012;69:292-300. http://dx.doi.org/10.1016/j.jsurg.2011.11.008

18. Papadakis MA, Teherani A, Banach MA, Knettler TR, Rattner SL, Stern DT, et al. Disciplinary action by medical boards and prior behavior in medical school. $\mathrm{N}$ Engl J Med. 2005;353:2673-82. http://dx.doi.org/10.1056/ NEJMsa052596

19. Chretien KC, Greysen SR, Chretien JP, Kind T. Online posting of unprofessional content by medical students. JAMA. 2009;302:1309-15. http://dx.doi.org/10.1001/jama. 2009.1387

20. Thompson LA, Black E, Duff P, Paradise N, Saliba H, Dawson K. Protected health information on social networking sites: Ethical and legal considerations. J Med Internet Res. 2011;13:8. http://dx.doi.org/10.2196/jmir.1590

21. Landman MP, Shelton J, Kauffmann RM, Dattilo JB. Guidelines for maintaining a professional compass in the era of social networking. J Surg Educ. 2010;67:381-6. http://dx.doi.org/10.1016/j.jsurg.2010.07.006

22. Wojtczak A. Profesionalismo médico, una problemática global. Educ Med. 2006;9:144-45. http://dx.doi.org/10.4321/ S1575-18132006000300009

23. Morales-Ruiz JC. Formación integral y profesionalismo médico: una propuesta de trabajo en el aula. Educ Med. 2009;12:73-82. http://dx.doi.org/10.4321/S157518132009000300003

24. Rodríguez JJ. Definición de profesión médica, profesional médico/a y profesionalismo médico. Educ Med. 2010;13:63-6. http://dx.doi.org/10.4321/S1575-18132010000200001

25. Golden JB, Sweeny L, Bush B, Carroll WR. Social networking and professionalism in otolaryngology residency applicants. Laryngoscope. 2012;122:1493-6. http://dx.doi. org/10.1002/lary.23388 
26. Ponce BA, Determann JR, Boohaker HA, Sheppard E, McGwin Jr G, Theiss S. Social networking profiles and professionalism issues in residency applicants. An original study-cohort study. J Surg. 2013;70:502-7. http://dx.doi. org/10.1016/j.jsurg.2013.02.005

27. Guseh JS, Brendel RW, Brendel DH. Medical professionalism in the age of online social networking. J Med Ethics. 2009;35:584-6. http://dx.doi.org/10.1136/jme.2009.029231

28. Shore R, Halsey J, Shah K, Crigger BJ, Douglas SP, Council on Ethical and Judicial Affairs of American Medical Association. Report of the AMA Council on Ethical and Judicial Affairs: Professionalism in the use of social media. J Clin Ethics. 2011;22:165-72.

29. British Medical Association. Using social media: Practical and ethical guidance for doctors and medical students. London: British Medical Association; 2011. Fecha de consulta: 10 de septiembre de 2014. Disponible en: http:// bma.org.uk/practical-support-at-work/ethics/ethics-a-to-z

30. Mansfield SJ, Morrison SG, Stephens HO, Bonning MA, Wang SH, Withers AH, et al. Social media and the medical profession. Med J Aust. 2011;194:642-4.

31. Organización Médica Colegial. Manual de estilo para médicos y estudiantes de medicina sobre el buen uso de redes sociales. Madrid: Consejo General de Colegios Oficiales de Médicos de España; 2014. Fecha de consulta: 9 de septiembre de 2014. Disponible en: http:// eticamedicarrss.com/2014/05/13/manual-de-estilo-paramedicos-y-estudiantes-de-medicina-sobre-el-buen-uso-deredes-sociales/.

32. Garnica K, Calero A. Caracterización del estudiante javeriano. Voces y rostros de jóvenes javerianos. Cali: Decanatura del Medio Universitario de la Pontificia Universidad Javeriana; 2000.

33. Aigneren M. La técnica de recolección de información mediante grupos focales. La Sociología en sus Escenarios. 2002;6:1-32.

34. Escobar J, Bonilla FI. Grupos focales: una guía conceptual y metodológica. Cuadernos Hispanoamericanos de Psicología. 2009;9:51-67.

35. Ministerio de Salud. Resolución 008430 del 4 de octubre. Resolución por la cual se establecen las normas científicas, técnicas y administrativas para la investigación en salud. Bogotá: 1993. Fecha de consulta: 27 de julio de 2015. Disponible en: http://www.urosario.edu.co/urosario files/a2/ a24fb07a-f561-4fcc-b611-affff4374bb7.pdf.

36. Asociación Médica Mundial. Principios éticos para las investigaciones médicas en seres humanos. Declaración de Helsinki. Finlandia: 1964. Fecha de consulta: 27 de julio de 2015.. Disponible en: http://www.urosario.edu.co/EMCS/ Documentos/investigacion/declaracion_helsinki/.
37. Chretien K, Azar J, Kind T. Physicians on Twitter. JAMA. 2011;305:566-8. http://dx.doi.org/10.1001/jama.2011.68

38. Lagu T, Greysen SR. Physician, monitor thyself: Professionalism and accountability in the use of social media. J Clin Ethics. 2011;22:187-90

39. Greysen SR, Kind T, Chretien KC. Online professionalism and the mirror of socialmedia. J Gen Intern Med. 2010;25: 1227-9. http://dx.doi.org/10.1007/s11606-010-1447-1

40. Greysen SR, Chretien KC, Kind T, Young A. Gross P. Physician violations of online professionalism and disciplinary actions: A national survey of state medical boards. JAMA. 2012;307:1141-2. http://dx.doi.org/10.1001/ jama.2012.330

41. McMahon JW. Professionalism in the use of social media. Council on Ethical and Judicial Affairs. Chicago: American Medical Association; 2011. p. 1-7.

42. Clyde JW, Domenech JM, Geiser C. Medical professionalism: An experimental look at physicians' Facebook profiles. Med Educ Online. 2014;19:23149-56. http://dx.doi. org/10.3402/meo.v19.23149

43. Farnan JM, Snyder-Sulmasy L, Worster BK, Chaudhry HJ, Rhyne JA, Arora VM, et al. Online medical professionalism: Patient and public relationships: Policy statement from the American College of Physicians and the Federation of State Medical Boards. Ann Intern Med. 2013;158:620-7. http:// dx.doi.org/10.7326/0003-4819-158-8-201304160-00100

44. Congreso de la República de Colombia. Ley 23/1981 del 27 de febrero. Por la cual se dictan normas en materia de ética médica. Diario Oficial de la República de Colombia, No. 35711. Bogotá, D.C.: Congreso de la República; 1981.

45. Congreso de la República de Colombia. Resolución 1995 del 08 de julio de 1999. Por la cual se establecen normas para el manejo de la Historia Clínica. Diario Oficial de la República de Colombia, No. 43655. Bogotá, D.C.: Congreso de la República; 1999.

46. von Muhlen M, Ohno-Machado L. Reviewing social media use by clinicians. J Am Med Inform Assoc. 2012;19:777-81. http://dx.doi.org/10.1136/amiajnl-2012-000990

47. Farooqi $\mathbf{H}$, Patel $\mathbf{H}$, Aslam $\mathbf{H M}$, Ansari IQ, Khan $\mathbf{M}$, Iqbal $\mathbf{N}$, et al. Effect of Facebook on the life of medical university students. Int Arch Med. 2013;6:40-8. http://dx.doi. org/10.1186/1755-7682-6-40

48. Brynolf A, Johansson S, Appelgren E, Lynoe N, Edstedt AK. Virtual colleagues, virtually colleagues-physicians' use of Twitter: A population-based observational study. BMJ. 2013;3:2988-95. http://dx.doi.org/10.1136/bmjopen-2013002988 